\title{
Alignment-resolved $\mathrm{O}_{2}$ scattering from highly oriented pyrolytic graphite and $\mathrm{LiF}(001)$ surfaces
}

\author{
Mitsunori Kurahashi ${ }^{1}$ and Takahiro Kondo ${ }^{2}$ \\ ${ }^{1}$ National Institute for Materials Science, 1-2-1 Sengen, Tsukuba, Ibaraki 305-0047, Japan \\ ${ }^{2}$ Faculty of Pure and Applied Sciences, University of Tsukuba, 1-1-1 Tennoudai, Tsukuba, Ibaraki 305-8573, Japan
}

(Received 14 September 2018; revised manuscript received 11 December 2018; published 25 January 2019)

\begin{abstract}
We present an alignment-resolved $\mathrm{O}_{2}$ scattering experiment showing that the alignment of an incident homonuclear diatomic molecule strongly influences the angular distribution of scattered molecule, the energy loss during the collision, and the trapping probability. Clear surface rainbow scattering has been observed on $\mathrm{LiF}(001)$ when $\mathrm{O}_{2}$ is scattered along the [110] azimuth with its axis perpendicular to the scattering plane. The results can be understood based on the alignment dependence of the surface corrugation felt by an incident molecule. The fact that $\mathrm{O}_{2}$ trapping probability on a highly oriented pyrolytic graphite surface is higher for end-on collision has been associated with the observed larger energy loss for this geometry.
\end{abstract}

DOI: 10.1103/PhysRevB.99.045439

\section{INTRODUCTION}

State-resolved molecular beam-surface scattering experiments, which specify the geometry and internal states of incident and/or scattered molecules, provide valuable information not only on the geometrical effect in the molecule-surface interaction, but also on the mechanism of energy dissipation of impinging molecules necessary to result in trapping or chemisorption [1-6]. The geometrical effect here is usually classified into two categories. One is the orientation effect and the other is the alignment effect. The effect of orientation, i.e., the head-to-tail direction of an incident heteronuclear molecule, on its interaction with surfaces has been investigated for several polar molecule-surface systems [7-15]. It has been shown that, for NO scattering from flat metallic surfaces, the observed orientation dependence in the angular distribution and the rotational excitation of scattered molecules [10-13] can be understood based on the orientation dependent molecule-surface interaction potential $[14,15]$.

In contrast, experimental studies concerning the alignment effect, which is associated with the difference between "side-on" and "end-on" collisions, have been limited to the chemisorption studies [16-18] and that for ion-surface interaction [19]. Its effect on nonreactive molecule-surface scattering however remains mostly unexplored. Unlike the scattering of heteronuclear molecules where the orientation effect is dominant, alignment would be the key factor in the scattering of heavy homonuclear molecules, such as $\mathrm{O}_{2}$, $\mathrm{N}_{2}$, etc. These molecules have an elongated shape and their dimensions [20] are often comparable to the spatial period of surface corrugation. It is therefore expected that, depending on the alignment relative to the surface, the direction of the forces acting on the incident molecule and/or the surface corrugation felt by it can be largely different, possibly affecting the angular distribution of scattered molecules and the energy dissipation processes. This may be closely related to the fact that non-state-resolved polyatomic molecule-surface scattering experiments on a $\operatorname{LiF}(100)$ surface show structureless angular distributions $[21,22]$, while atom [22,23] or small spherical molecule [24] scattering on it exhibits clear surface rainbow scattering. Kondo et al. [22], based on the simulation with the ellipsoid washboard model, have associated this with the averaged contribution of differently aligned molecules contained in the randomly oriented beam used. To the best of our knowledge, however, surface scattering experiments with a beam of aligned heavy homonuclear molecules necessary to clarify this issue have not been reported yet.

In this paper, we present experimental evidence showing that the alignment of $\mathrm{O}_{2}$ affects the angular distribution of scattered molecule, the trapping probability, and the energy dissipation efficiency. We also demonstrate that the surface rainbow scattering does appear if the collision occurs at a geometry where $\mathrm{O}_{2}$ can feel the surface corrugation effectively.

\section{EXPERIMENT}

The alignment-resolved $\mathrm{O}_{2}$ scattering experiments have been conducted with a single spin-rotational state-selected $[(J, M)=(2,2)] \mathrm{O}_{2}$ beam [17,25]. The state-selected $\mathrm{O}_{2}$ beam was prepared by passing a supersonic seeded $\mathrm{O}_{2} / \mathrm{He}$ beam through a hexapole magnet. Here, the magnetic hexapolar field was used as a filter to transmit the $(2,2)$ state and reject others. The translational energy $\left(E_{0}\right)$ of the $\mathrm{O}_{2}(2,2)$ beam was controlled in the energy range of $0.08-0.25 \mathrm{eV}$ by adjusting the length of the hexapole and the velocity of the seeded $\mathrm{O}_{2}$ beam. The intensity of $\mathrm{O}_{2}$ molecules scattered or desorbed from the crystal, which is rotatable along the axis perpendicular to the scattering plane, was measured by a differentially pumped mass spectrometer equipped at a fixed angle of $45^{\circ}$ relative to the incidence beam [Fig. 1(a)]. The sum of the incidence angle $\left(\theta_{i}\right)$ and scattering angle $\left(\theta_{f}\right)$ relative to the surface normal is therefore $45^{\circ}$. Since the rotational quantum numbers for the $(J, M)=(2,2)$ state are specified to be $\left(K, M_{K}\right)=(1,1)$, the $\mathrm{O}_{2}$ axis angular distribution function is given by $\left|Y_{1}^{1}\right|^{2}\left(=\frac{3}{8 \pi} \sin ^{2} \alpha\right)$, where $\alpha$ is the polar angle of the molecular axis relative to the defining magnetic field $(H)$ direction $[16,17,25]$. 
(a)

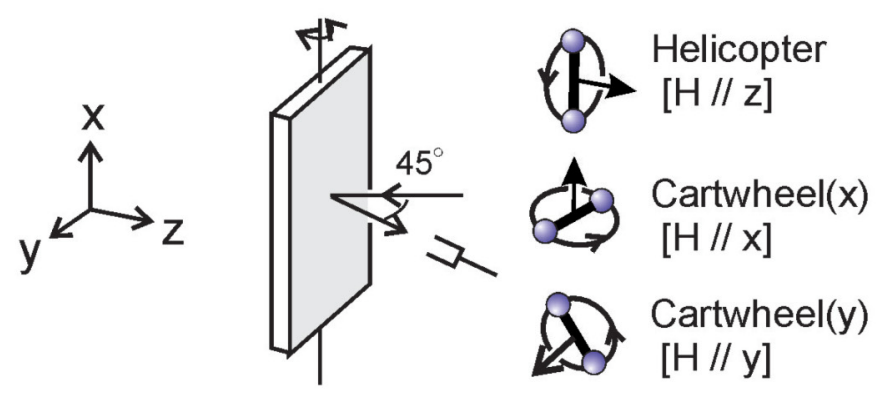

(b)

(c)

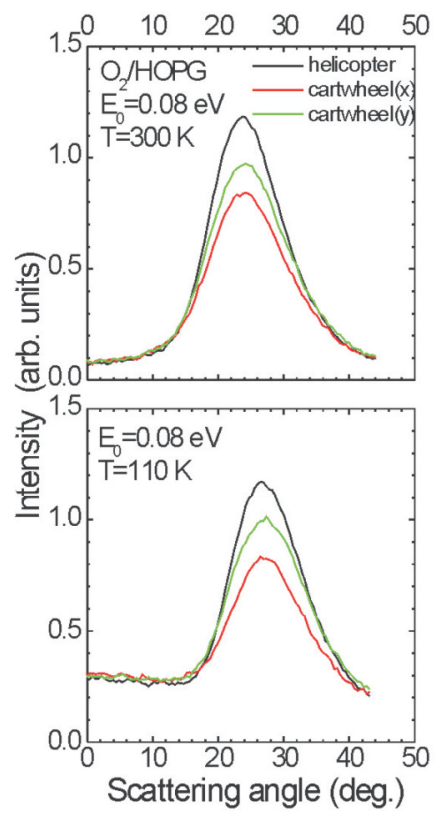

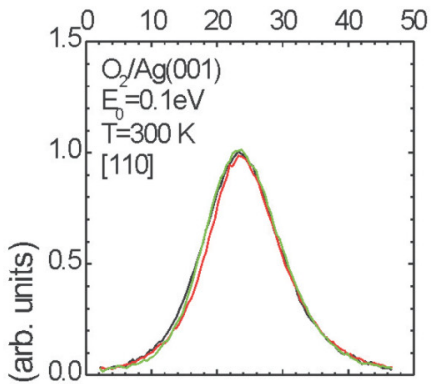

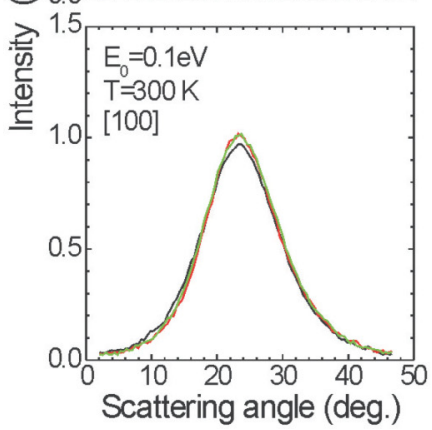

(d)

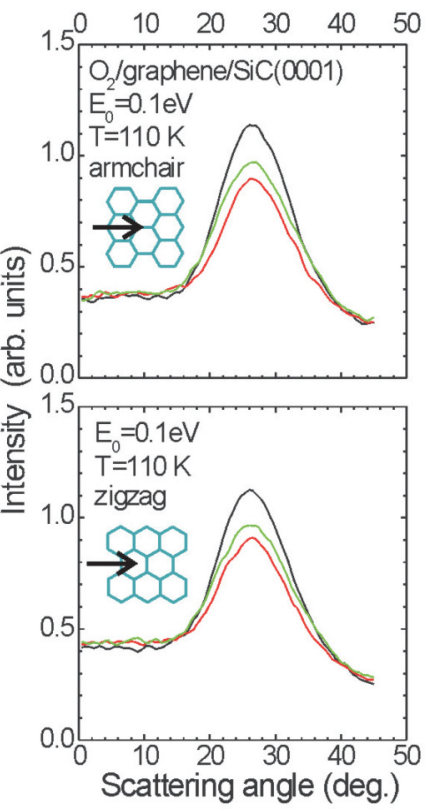

FIG. 1. (a) Three different geometries of incident $\mathrm{O}_{2}$ used for the scattering experiment. (b)-(d) The angular intensity distributions of $\mathrm{O}_{2}$ molecules scattered from (b) HOPG, (c) $\mathrm{Ag}(001)$, and (d) graphene/SiC $(0001)$ surfaces. The translational energy, the sample temperature, and the azimuth along which the scattering was monitored are shown in the figures. The scattering spectra are normalized to the main peak intensity at $20^{\circ}-30^{\circ}$ for random orientation, which is the average for the three geometries.

The scattering experiments were conducted at helicopter and two nonequivalent cartwheel geometries shown in Fig. 1(a). In helicopter geometry, where $H$ is along the surface normal ( $z$ direction), the molecular axis of incident $\mathrm{O}_{2}$ is mainly parallel to the surface. Two cartwheel geometries, where $H$ is parallel to the surface, contain contributions of perpendicular molecules, but these two are different in that the rotational axis is perpendicular (parallel) to the scattering plane in cartwheel $(x)$ [cartwheel $(y)$ ] geometries. The $H$ direction was changed following the incidence angle scan during the measurement. The fluctuation of the beam and detector conditions was less than $1 \%$. We note that the rotational energy for the $\mathrm{O}_{2}(2,2)$ state is $0.36 \mathrm{meV}$ [17], which is much lower than the $E_{0}$ values used in the present study. This means that, since the time scale for translational motion is much faster than a rotational period, incident rotational motion is almost frozen during the molecule's final approach to the surface. In such a situation, alignment of incident $\mathrm{O}_{2}$ is much more important than its rotational motion.
A highly oriented pyrolytic graphite (HOPG) substrate (ZYA-grade, Panasonic), after being cleaved in the air, was annealed up to $873 \mathrm{~K}$ in the ultrahigh vacuum chamber to prepare its clean surface. The epitaxial graphene was prepared on a Si-terminated (0001) face of $6 \mathrm{H}-\mathrm{SiC}$ by heating the substrate at $1400{ }^{\circ} \mathrm{C}$ for $10 \mathrm{~min}$. A low energy electron diffraction (LEED) pattern of the surface (Fig. 2) was similar to that reported in Ref. [26]. The ratio of the intensity of C-270 eV and Si-90 eV Auger peaks at $3 \mathrm{keV}$ incident energy was 13:1, from which the thickness of the graphite layers was estimated to be about 3 monolayers. A $\mathrm{LiF}(001)$ substrate cleaved from a bulk single crystal block was annealed at $723 \mathrm{~K}$ for a few hours to obtain a well-ordered surface, which has been checked by low energy electron diffraction measurements. A clean $\mathrm{Ag}(001)$ surface was prepared by repeating the annealing at $700 \mathrm{~K}$ and $\mathrm{Ar}^{+}$sputtering at $500 \mathrm{eV}$.

The intensity of scattered $\mathrm{O}_{2}$ or He was monitored with a lock-in technique by modulating the primary beam with a $50 \%$ duty-cycle chopper. The ion source of the mass spectrometer was magnetically shielded to avoid the effect of the change in 


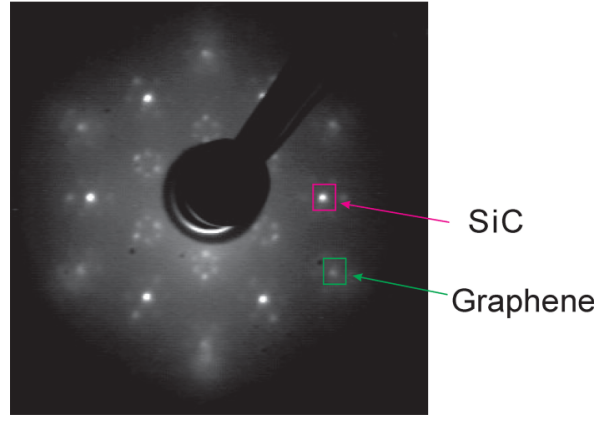

FIG. 2. Low energy electron diffraction pattern from graphene/ $\mathrm{SiC}(0001)$ measured at the beam energy of $100 \mathrm{eV}$.

the defining magnetic field direction on the ionization yield. Since a small amount of $\mathrm{O}_{2}$ was accumulated during the scattering measurement on $\operatorname{Ag}(001)$, the $\operatorname{Ag}(001)$ substrate was annealed shortly at $700 \mathrm{~K}$ after each measurement to recover the clean surface.

\section{RESULTS AND DISCUSSIONS}

\section{A. Direct inelastic scattering of $\mathrm{O}_{2}$ from HOPG}

Figure 1(b) shows the angular intensity distribution of $\mathrm{O}_{2}$ molecules scattered or desorbed from an HOPG surface measured at the three geometries. The peak at $\theta_{f}=20^{\circ}-30^{\circ}$ is associated with direct inelastic scattering following the previous $\mathrm{O}_{2}$ scattering study on HOPG [27]. The peak intensity depends strongly on the geometry, and this dependency is larger at lower temperature. It also shows a broad backgroundlike feature, which has a higher intensity at lower temperature and at around the surface normal $\left(\theta_{f}=0^{\circ}\right)$. As will be shown later, it attenuates steeply with increasing $E_{0}$. This feature can therefore be attributed to trapping followed by desorption.

To see the effect of surface corrugation and orientation of graphite domain relative to the scattering plane, we conducted the same experiments on $\mathrm{Ag}(001)$ [Fig. 1(c)] and an epitaxial graphene prepared on $\mathrm{SiC}(0001)$ [Fig. 1(d)]. The $\mathrm{O}_{2}$ scattering experiments from $\operatorname{Ag}(001)$, which is more flat, along the [110] and [100] azimuths show much weaker geometry dependences, suggesting that surface corrugation would be the main cause of the geometry dependence observed for $\mathrm{O}_{2} / \mathrm{HOPG}$. The $\mathrm{O}_{2}$ scattering experiments on the graphene along armchair and zigzag directions show similar geometry dependencies, indicating that the orientation of the graphite domain relative to the scattering plane has little effect on the observed geometry dependence. The geometry dependence observed for the graphene is weaker than that for HOPG, which is probably due to the poorer quality of the present graphene surface. Actually, although the angular width of the specularly reflected $\mathrm{He}$ intensity for $\mathrm{HOPG}$ was $\sim 1.5^{\circ}$, which is close to the acceptance angle of the detector aperture, it was $\sim 4^{\circ}$ for the graphene. We therefore use the results for HOPG in the following analysis.

To clarify the origin of the observed alignment effect, the measured scattering/desorption spectra were decomposed into those for the $\mathrm{O}_{2}$ axis angular distribution functions $\rho_{x}=\frac{3}{4 \pi} \sin ^{2} \theta \cos ^{2} \phi, \rho_{y}=\frac{3}{4 \pi} \sin ^{2} \theta \sin ^{2} \phi$, and $\rho_{z}=\frac{3}{4 \pi} \cos ^{2} \theta$ [Fig. 3(a)], where $\theta$ and $\phi$ are the polar and azimuth angles (a)

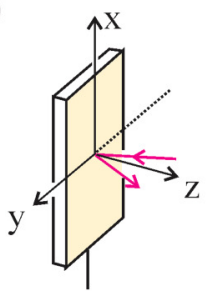

(c)

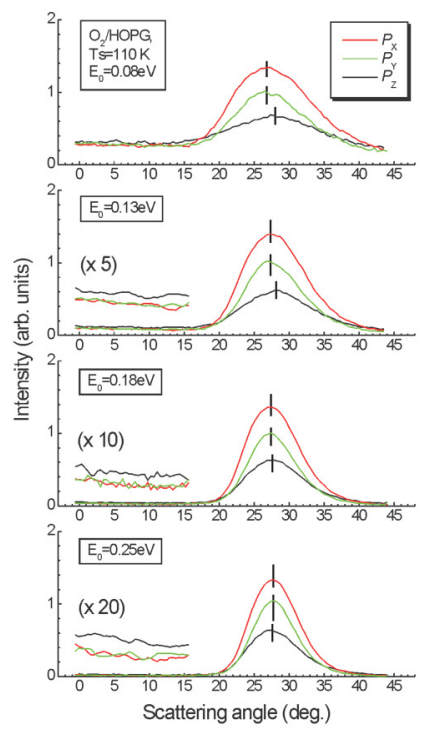

(e)

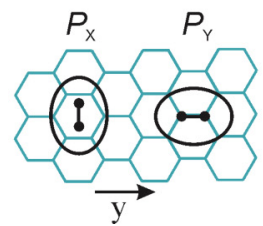

(b)

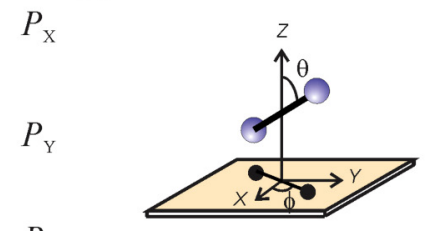

(d)

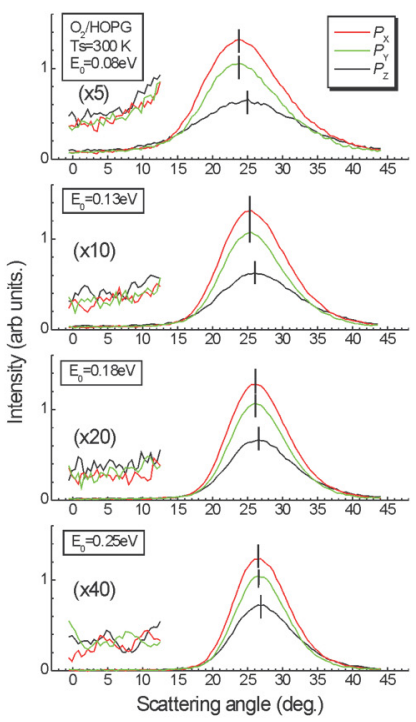

(f)

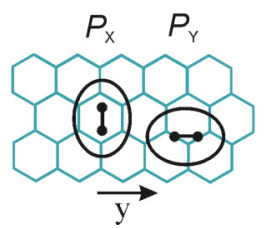

FIG. 3. (a) $\mathrm{O}_{2}$ axis angular distribution functions for $P_{X}, P_{Y}$, and $P_{Z}$ geometries. $\mathrm{O}_{2}$ is scattered within the $y z$ plane. (b) The definition of the angles representing the $\mathrm{O}_{2}$ axis direction. (c),(d) The angular intensity distributions of $\mathrm{O}_{2}$ scattered from an HOPG surface at (c) $110 \mathrm{~K}$ and (d) $300 \mathrm{~K}$ derived for the $P_{X}, P_{Y}$, and $P_{Z}$ geometries. The scattering spectra are normalized to the main peak intensity at $20^{\circ}-30^{\circ}$ for random orientation. The low $\theta_{f}$ part of the spectra that have been rescaled and smoothed by the simple moving average are also shown. (e),(f) Comparison of the $P_{X}$ and $P_{Y}$ geometries for the $\mathrm{O}_{2}$ scattering from graphite along (e) armchair and (f) zigzag directions.

of $\mathrm{O}_{2}$ axis relative to the $x y z$ coordinates [Fig. 3(b)]. The $\mathrm{O}_{2}$ axis is distributed mainly along $x, y$, and $z$ axes in $\rho_{x}$, $\rho_{y}$, and $\rho_{z}$, respectively, and these geometries are denoted as $P_{X}, P_{Y}$, and $P_{Z}$ [Fig. 3(a)]. Linear combinations of the measured spectra [Fig. 1(b)] allow this decomposition since the distribution functions for the helicopter, cartwheel $(x)$, and cartwheel $(y)$ geometries are equal to $\frac{1}{2}\left(\rho_{x}+\rho_{y}\right), \frac{1}{2}\left(\rho_{y}+\rho_{z}\right)$, and $\frac{1}{2}\left(\rho_{x}+\rho_{z}\right)$, respectively.

The spectra derived for $P_{X}, P_{Y}$, and $P_{Z}$ geometries [Figs. 3(c) and 3(d)] show the $\mathrm{O}_{2}$ alignment effect more clearly. First, the intensity of the in-plane direct inelastic scattering is in the order of $I\left(P_{X}\right)>I\left(P_{Y}\right)>I\left(P_{Z}\right)$. Second, its peak width is in the order of $\Delta \theta_{f}\left(P_{Z}\right)>\Delta \theta_{f}\left(P_{X}\right)>\Delta \theta_{f}\left(P_{Y}\right)$. 
TABLE I. Position $\left(\theta_{p}\right)$, the full width at half maximum $\left(\Delta \theta_{f}\right)$, and the relative intensity $\left(I_{D}\right)$ of the peak due to the direct inelastic scattering of $\mathrm{O}_{2}$ from $\mathrm{HOPG}$ at $110 \mathrm{~K}$ for $P_{X}, P_{Y}$, and $P_{Z}$ geometries. The relative intensity of the trapping followed by desorption $\left(I_{T D}\right)$ is also shown.

\begin{tabular}{|c|c|c|c|c|c|c|c|c|c|c|c|c|}
\hline \multirow[b]{2}{*}{$E_{0}(\mathrm{eV})$} & \multicolumn{4}{|c|}{$P_{X}$} & \multicolumn{4}{|c|}{$P_{Y}$} & \multicolumn{4}{|c|}{$P_{Z}$} \\
\hline & $\theta_{p}(\mathrm{deg})$ & $\Delta \theta_{f}(\mathrm{deg})$ & $I_{D}$ & $I_{T D}$ & $\theta_{p}(\mathrm{deg})$ & $\Delta \theta_{f}(\mathrm{deg})$ & $I_{D}$ & $I_{T D}$ & $\theta_{p}(\mathrm{deg})$ & $\Delta \theta_{f}(\mathrm{deg})$ & $I_{D}$ & $I_{T D}$ \\
\hline 0.08 & 26.8 & 12.3 & 1.10 & 0.25 & 26.7 & 10.8 & 0.74 & 0.26 & 27.9 & 12.5 & 0.37 & 0.30 \\
\hline 0.13 & 27.5 & 10.1 & 1.33 & 0.084 & 27.2 & 9.2 & 0.92 & 0.085 & 28.2 & 10.7 & 0.50 & 0.11 \\
\hline 0.18 & 27.3 & 8.7 & 1.35 & 0.032 & 27.3 & 7.9 & 0.95 & 0.034 & 27.6 & 9.3 & 0.59 & 0.045 \\
\hline 0.25 & 27.7 & 7.9 & 1.32 & 0.018 & 27.7 & 7.2 & 1.01 & 0.019 & 27.5 & 8.5 & 0.60 & 0.028 \\
\hline
\end{tabular}

These observed tendencies are similar over all the experimental range of energies used in this work. Third, the peak position for end-on collision ( $P_{Z}$ geometry) is shifted by $\sim 1^{\circ}$ to higher $\theta_{f}$ than that for side-on collision $\left(P_{X}\right.$ and $P_{Y}$ geometries) at $E_{0}=0.08 \mathrm{eV}$. This peak position shift decreases with increasing $E_{0}$ and almost disappears at $E_{0}=$ $0.25 \mathrm{eV}$. This behavior is observed both at $110 \mathrm{~K}$ and $300 \mathrm{~K}$. Tables I and II summarize the geometry dependence of the peak position, width and intensity of $\mathrm{O}_{2}$ scattering/desorption from $\mathrm{HOPG}$ at $110 \mathrm{~K}$ and $300 \mathrm{~K}$, respectively. The parameters shown were derived by fitting the spectra to a Gaussian function $I_{D} \exp \left[-\left(\theta_{f}-\theta_{p}\right)^{2} / 2 w^{2}\right]+I_{T D}$. The values of $I_{D}$ and $I_{T D}$ reflect the relative intensities of the direct inelastic scattering and the trapping followed by desorption, respectively. $I_{T D}$ values at $300 \mathrm{~K}$ are not shown since the contribution of the trapping followed by desorption is not clear at $300 \mathrm{~K}$.

The alignment dependence of in-plane direct inelastic scattering from HOPG can be understood based on the fact that surface corrugation felt by an incident $\mathrm{O}_{2}$ molecule depends on the $\mathrm{O}_{2}$ alignment relative to the surface. On an HOPG surface, valence electron density is higher above the $\mathrm{C}-\mathrm{C}$ bond of a benzene ring than above the center of the ring, causing the corrugation of the $\mathrm{O}_{2}$-surface interaction potential. Here, the distance between neighboring benzene ring is $\sim 2.5 \AA$, while $\mathrm{O}_{2}$ is an ellipsoidal molecule with a length of $4.2 \AA$ and a width of $3.2 \AA$, which have been estimated based on the computed charge analysis [20].

To discuss the origin of the geometry dependence of the in-plane direct scattering, two types of corrugation have to be considered. If the corrugation perpendicular to the scattering plane is large, the probability of out-of-plane scattering is high, making in-plane scattering intensity lower. If the corrugation within the scattering plane is large, its angular intensity distribution becomes wider. We first consider the case of end-on collision ( $P_{Z}$ geometry). Since the end of an $\mathrm{O}_{2}$ molecule collides with the surface, both types of surface corrugation are larger than those for side-on collision. This accounts for the lowest in-plane direct scattering intensity and the largest peak width for $P_{Z}$ geometry [Figs. 3(c) and $3(\mathrm{~d})]$.

Second, we discuss the different scattering behaviors between $P_{X}$ and $P_{Y}$ geometries. Here, it is important to note that the longitudinal dimension of $\mathrm{O}_{2}$ is considerably larger than the diameter of the benzene ring of graphite, while the lateral dimension of $\mathrm{O}_{2}$ is comparable with it [Figs. 3(e) and 3(f)]. $\mathrm{O}_{2}$ therefore would not feel a large corrugation along the longitudinal direction but feel a larger corrugation along the transverse direction. It is therefore expected that, in $P_{X}$ geometry where the $\mathrm{O}_{2}$ axis is perpendicular to the scattering plane, $\mathrm{O}_{2}$ feels a smaller corrugation perpendicular to the scattering plane but a larger corrugation within the scattering plane than for $P_{Y}$ geometry. This would cause a larger intensity but a broader width of the in-plane scattering peak for $P_{X}$ geometry, which is consistent with the observed behaviors. Considering the size of $\mathrm{O}_{2}$ relative to the benzene ring and the relatively isotropic shape of the benzene ring, similar corrugation difference is expected both along the armchair and zigzag directions [Figs. 3(e) and 3(f)]. This expectation is supported by the fact that similar geometry dependence was observed for $\mathrm{O}_{2}$ scattering along these two directions on the graphene [Fig. 1(d)]. The presence of the similar geometry dependence for the $\mathrm{O}_{2} / \mathrm{HOPG}$ system suggests that the orientation of the graphite domain relative to the scattering plane has little effect on the difference in corrugation between $P_{X}$ and $P_{Y}$ geometries.

Raukema et al. [28] have shown that the intensity of inplane $\mathrm{O}_{2}$ scattering from $\operatorname{Ag}(110)$ along [1iㅣ azimuth is much lower than that along [001] azimuth. This has been attributed to the fact that $\mathrm{O}_{2}$ is more easily scattered to the outof-plane direction if $\mathrm{O}_{2}$ is incident along the row of surface corrugation. Their explanation is similar to our discussion which associates in-plane scattering intensity with the effective surface corrugation perpendicular to the scattering plane.

TABLE II. Parameters for the $\mathrm{O}_{2}$ scattering from $\mathrm{HOPG}$ at $300 \mathrm{~K}$.

\begin{tabular}{|c|c|c|c|c|c|c|c|c|c|}
\hline \multirow[b]{2}{*}{$E_{0}(\mathrm{eV})$} & \multicolumn{3}{|c|}{$P_{X}$} & \multicolumn{3}{|c|}{$P_{Y}$} & \multicolumn{3}{|c|}{$P_{Z}$} \\
\hline & $\theta_{p}(\mathrm{deg})$ & $\Delta \theta_{f}(\mathrm{deg})$ & $I_{D}$ & $\theta_{p}(\mathrm{deg})$ & $\Delta \theta_{f}(\operatorname{deg})$ & $I_{D}$ & $\theta_{p}(\mathrm{deg})$ & $\Delta \theta_{f}(\operatorname{deg})$ & $I_{D}$ \\
\hline 0.13 & 25.3 & 11.1 & 1.25 & 25.3 & 10.6 & 1.01 & 26.0 & 13.1 & 0.56 \\
\hline 0.18 & 26.2 & 9.9 & 1.25 & 26.2 & 9.5 & 1.03 & 26.4 & 11.0 & 0.63 \\
\hline 0.25 & 26.6 & 9.2 & 1.22 & 26.7 & 8.7 & 1.02 & 26.8 & 10.2 & 0.69 \\
\hline
\end{tabular}


(a)
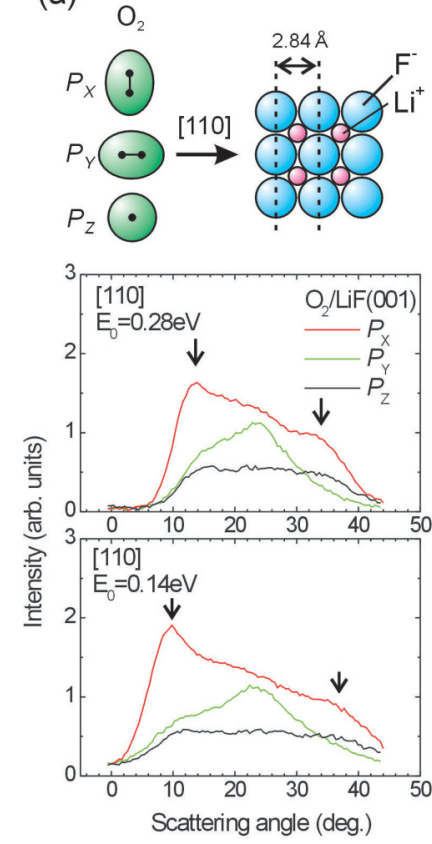

(b)
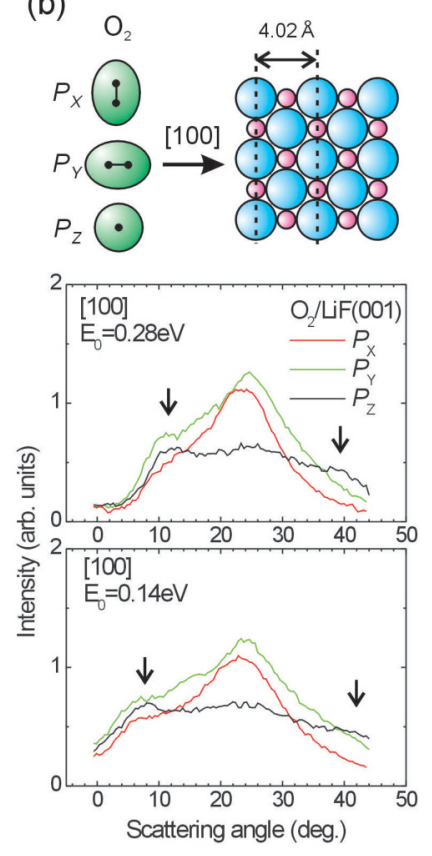

FIG. 4. Angular intensity distributions of $\mathrm{O}_{2}$ molecules scattered from $\mathrm{LiF}(001)$ surface along (a) [110] and (b) [100] azimuths derived for $P_{X}, P_{Y}$, and $P_{Z}$ geometries. The sample temperature is $300 \mathrm{~K}$. Upper and lower panels show the spectra at $E_{0}=0.28 \mathrm{eV}$ and $0.14 \mathrm{eV}$, respectively. The scattering spectra are normalized to the intensity of the peak at $20^{\circ}-30^{\circ}$ for random orientation. The arrows indicate the position of the peaks due to the rainbow scattering.

\section{B. Rainbow scattering of $\mathrm{O}_{2}$ from $\operatorname{LiF}(100)$}

To see the effect of surface corrugation on the direct scattering more clearly, we investigated the alignment dependence in $\mathrm{O}_{2}$ scattering from $\mathrm{LiF}(001)$. Figures $4(\mathrm{a})$ and $4(\mathrm{~b})$ show the alignment dependent $\mathrm{O}_{2}$ scattering from $\mathrm{LiF}(001)$ along [110] and [100] azimuths, respectively. Clear peaks associated with surface rainbow scattering appear at $\theta_{f}=9^{\circ}$ and $36^{\circ}$ at $E_{0}=$ $0.14 \mathrm{eV}$ when $\mathrm{O}_{2}$ is scattered along the [110] azimuth with its axis perpendicular to the scattering plane ( $P_{X}$ geometry). Intuitively, these rainbow peaks can be understood as the two scattering components coming from the collision with the uphill and downhill part of the surface local corrugation. The rainbow peaks are observed at the same angles also in case of end-on collision although their intensities are much lower. The two peaks approach each other with increasing $E_{0}$ similar to the case of $\mathrm{Ar}$ scattering from $\operatorname{LiF}(001)[22,29,30]$. In contrast, when the $\mathrm{O}_{2}$ axis is parallel to the scattering plane $\left(P_{Y}\right.$ geometry), the rainbow peaks are strongly attenuated and a peak attributed to the specular scattering $\left(\theta_{f} \sim 24^{\circ}\right)$ is dominant.

These behaviors can be understood based on the $\mathrm{O}_{2}$ alignment dependence of corrugation. The surface has a square lattice and $\mathrm{F}^{-}$ions with which $\mathrm{O}_{2}$ mainly interacts are aligned along the [110] and [1/10] directions. When $\mathrm{O}_{2}$ is scattered along the [110] azimuth with its axis parallel to the [1 $\overline{1} 0]$ direction, it can feel the surface corrugation strongly, resulting in the surface rainbow scattering similar to that for the scattering of $\mathrm{Ne}$ [23] and $\mathrm{Ar}$ [22]. However, when the $\mathrm{O}_{2}$ axis is

parallel to the scattering plane, $\mathrm{O}_{2}$ cannot feel the corrugation created by surface $\mathrm{F}^{-}$ions because of its elongated shape, making the specular scattering dominant. In case of end-on collision, since $\mathrm{O}_{2}$ feels the corrugation within the scattering plane, the surface rainbow scattering occurs. Its intensity is however low because $\mathrm{O}_{2}$ also feels a large corrugation perpendicular to the scattering plane and the out-of-plane scattering probability is high. A small specular scattering peak is seen even in the spectra for $P_{X}$ geometry. This may be due to the fact that the $\mathrm{O}_{2}$ axis angular distribution function has a finite angular width.

Figure 4(b) shows that, unlike the $\mathrm{O}_{2}$ scattering along the [110] azimuth, the rainbow scattering along the [100] azimuth is much weaker and the specular scattering peak is dominant both in $P_{X}$ and $P_{Y}$ geometries. This would be due to the fact that, since the $\mathrm{O}_{2}$ axis is $45^{\circ}$ relative to the row of surface $\mathrm{F}^{-}$ions, $\mathrm{O}_{2}$ cannot feel the surface corrugation effectively. Rainbow scattering appears in the case of $P_{Z}$ geometry since $\mathrm{O}_{2}$ feels the corrugation created by $\mathrm{F}^{-}$ions along the [100] azimuth similar to the case of $\operatorname{Ar} / \operatorname{LiF}(001)$ [22]. The larger separation of the rainbow peaks than along the [110] azimuth reflects the larger distance between neighboring $\mathrm{F}^{-}$ions along the [100] azimuth [22].

\section{Energy loss and trapping followed by desorption}

The alignment dependence in the energy loss during the $\mathrm{O}_{2}$-surface collision can be discussed based on the peak position of direct scattering and the trapping probability. The direct scattering peak is located at around $\theta_{f}=27^{\circ}\left(\theta_{i}=18^{\circ}\right)$ at $110 \mathrm{~K}$ [Fig. 3(c)]. Assuming parallel momentum conservation, we can estimate the loss of translational energy to be $\sim 54 \%$. The fact that the peak position for end-on collision is shifted by $\sim 1^{\circ}$ from that for side-on collision at $E_{0}=0.08 \mathrm{eV}$ means that the energy loss is $7 \%$ larger for end-on collision. The decrease of this peak shift with increasing $E_{0}$ suggests that the attractive part of the $\mathrm{O}_{2}$-graphite surface interaction potential, which becomes more important for collisions at lower $E_{0}$, plays an important role in the energy loss process and its geometry dependence causes the observed peak position difference. It might be possible that $\mathrm{O}_{2}$ approaches the surface more closely and feels a deeper potential well in case of end-on collision. It has been predicted that the rotational excitation of NO is strongly influenced by the attractive part of the interaction potential [14].

Figure 3(c) also shows the alignment dependence of the trapping followed by desorption, the contribution of which is seen at low $\theta_{f}$ region. Its intensity decreases steeply with increasing $E_{0}$. Its alignment dependence, although it is not so significant at $E_{0}=0.08 \mathrm{eV}$, becomes more clear at higher $E_{0}$. The spectra for end-on collision $\left(P_{Z}\right.$ geometry) show higher intensities than for the side-on collision $\left(P_{X}, P_{Y}\right)$, while those for $P_{X}$ and $P_{Y}$ geometries exhibit similar intensities. Since thermal $\mathrm{O}_{2}$ does not stick on HOPG at temperatures $>60 \mathrm{~K}$ [31], it would be reasonable to assume that the intensity of this feature is proportional to the trapping probability $\left(P_{\text {trap }}\right)$. Its intensity is much lower at $300 \mathrm{~K}$ [Fig. 3(d)].

The larger energy loss observed for end-on collision is consistent with the higher $P_{\text {trap }}$ for this geometry [Fig. 3(c)] because efficient energy loss makes trapping easier. The 
decrease of $P_{\text {trap }}$ with increasing $E_{0}$ apparently correlates with that of the peak shift of the direct scattering mentioned above. But, these two quantities may not be directly coupled because similar peak shift with $E_{0}$ has been observed also at $300 \mathrm{~K}$, where trapping is much less efficient [Fig. 3(d)]. The larger steric effect in trapping at higher $E_{0}$ [Fig. 3(c)] may be understood as follows. $P_{\text {trap }}$ decays with $E_{0}$ more gradually in the case of end-on collision because $\mathrm{O}_{2}$ can lose translational energy more efficiently. Therefore, although $P_{\text {trap }}$ is similarly high at low $E_{0}$, its ratio between the cases of end-on and side-on collision becomes larger with increasing $E_{0}$.

\section{CONCLUSIONS}

The present alignment-resolved scattering/desorption experiments have clarified that the geometry of an incident homonuclear molecule greatly affects the angular distribu- tion of the scattered molecule, the trapping probability, and the energy dissipation during the molecule-surface collision. Surface corrugation seen by an incident molecule has been shown to be the key to understanding the angular distribution of scattered molecules. Internal state analysis of scattered molecule and/or its theoretical simulation would be useful for further discussions about the origin of the observed geometry dependent energy loss.

\section{ACKNOWLEDGMENTS}

We are thankful to Prof. Inaki Juaristi, Prof. Maite Alducin, Prof. Daniel Farias, Dr. Masanori Yata, and Dr. Hirokazu Ueta for fruitful discussions, and to the anonymous reviewers for helpful comments on an earlier version of this paper. This work was supported by JSPS KAKENHI Grants No. 25286060, No. 16H03874, and No. 16K13691.
[1] A. W. Kleyn, Chem. Soc. Rev. 32, 87 (2003).

[2] G. O. Sitz, Rep. Prog. Phys. 65, 1165 (2002).

[3] J. Werdecker, M. E. van Reijzen, B. J. Chen, and R. D. Beck, Phys. Rev. Lett. 120, 053402 (2018).

[4] O. Godsi, G. Corem, Y. Alkoby, J. T. Cantin, R. V. Krems, M. F. Somers, J. Meyer, G. J. Kroes, T. Maniv, and G. Alexandrowicz, Nat. Commun. 8, 15357 (2017).

[5] P. R. Shirhatti, I. Rahinov, K. Golibrzuch, J. Werdecker, J. Geweke, J. Altschäffel, S. Kumar, D. J. Auerbach, C. Bartels, and A. M. Wodtke, Nat. Chem. 10, 592 (2018).

[6] C. Steinsiek, P. R. Shirhatti, J. Geweke, J. A. Lau, J. Altschäffel, A. Kandratsenka, C. Bartels, and A. M. Wodtke, J. Phys. Chem. C 122, 18942 (2018).

[7] T. J. Curtiss, R. S. Mackay, and R. B. Bernstein, J. Chem. Phys. 93, 7387 (1990).

[8] M. Okada, Chem. Rec. 14, 775 (2014).

[9] N. Bartels, K. Golibrzuch, C. Bartels, L. Chen, D. J. Auerbach, A. M. Wodtke, and T. Schäfer, Proc. Natl. Acad. Sci. USA 110, 17738 (2013).

[10] E. W. Kuipers, M. G. Tenner, A. W. Kleyn, and S. Stolte, Phys. Rev. Lett. 62, 2152 (1989).

[11] M. G. Tenner, E. W. Kuipers, A. W. Kleyn, and S. Stolte, J. Chem. Phys. 94, 5197 (1991).

[12] A. W. Kleyn, Prog. Surf. Sci. 54, 407 (1997).

[13] A. W. Kleyn and T. C. M. Horn, Phys. Rep. 199, 191 (1991).

[14] C. W. Muhlhausen, L. R. Williams, and J. C. Tully, J. Chem. Phys. 83, 2594 (1985).

[15] R. J. W. E. Lahaye, S. Stolte, S. Holloway, and A. W. Kleyn, J. Chem. Phys. 104, 8301 (1996).

[16] M. Kurahashi and Y. Yamauchi, Phys. Rev. Lett. 110, 246102 (2013); 114, 016101 (2015).
[17] M. Kurahashi, Prog. Surf. Sci. 91, 29 (2016).

[18] L. Vattuone, L. Savio, F. Pirani, D. Cappelletti, M. Okada, and M. Rocca, Prog. Surf. Sci. 85, 92 (2010).

[19] J. N. Greeley, J. S. Martin, J. R. Morris, and D. C. Jacobs, Surf. Sci. 314, 97 (1994).

[20] R. F. W. Bader, W. H. Henneker, and P. E. Cade, J. Chem. Phys. 46, 3341 (1967).

[21] T. Kondo, T. Tomii, S. Yagyu, and S. Yamamoto, J. Vac. Sci. Technol. A 19, 2468 (2001).

[22] T. Kondo, H. S. Kato, T. Yamada, S. Yamamoto, and M. Kawai, J. Chem. Phys. 122, 244713 (2005).

[23] J. N. Smith, Jr., D. R. O'Keefe, H. Saltsburg, and R. L. Palmer, J. Chem. Phys. 50, 4667 (1969).

[24] A. C. Wight and R. E. Miller, J. Chem. Phys. 109, 1976 (1998).

[25] M. Kurahashi and Y. Yamauchi, Rev. Sci. Instrum. 80, 083103 (2009).

[26] C. Berger, Z. Song, T. Li, X. Li, A. Y. Ogbazghi, R. Feng, Z. Dai, A. N. Marchenkov, E. H. Conrad, P. N. First, and W. A. de Heer, J. Phys. Chem. B 108, 19912 (2004).

[27] J. Oh, T. Kondo, K. Arakawa, Y. Saito, W. W. Hayes, J. R. Manson, and J. Nakamura, J. Phys. Chem. A 115, 7089 (2011).

[28] A. Raukema, D. A. Butler, and A. W. Kleyn, J. Phys.: Condens. Matter 8, 2247 (1996).

[29] S. Miret-Artés and E. Pollak, Surf. Sci. Rep. 67, 161 (2012).

[30] The analysis with classical perturbation theory [29] has suggested that this rainbow peak approach comes from the $E_{0}$ dependent energy transfer from a projectile to a corrugated surface with attractive potential, not from the $E_{0}$ dependence of the corrugation [22].

[31] H. Ulbricht, G. Moos, and T. Hertel, Phys. Rev. B 66, 075404 (2002). 